\title{
OBSERVING GRB AFTERGLOWS, SNE AND THEIR HOST GALAXIES WITH THE 10.4 M GRAN TELESCOPIO CANARIAS (GTC)
}

\author{
J. Gorosabel ${ }^{1,2,3}$, A.J. Castro-Tirado ${ }^{1}$, A. de Ugarte Postigo ${ }^{1,4}$, \\ C.C. Thöne ${ }^{1}$, R. Sánchez-Ramírez ${ }^{1}$, D. Peréz-Ramírez ${ }^{5}$, J.C. Tello ${ }^{1}$, \\ M. Jelínek ${ }^{1}$ and S. Guziy ${ }^{1,6}$
}

\begin{abstract}
We summarize the results of our programs started in 2009 with the $10.4 \mathrm{~m}$ Gran Telescopio Canarias (GTC) telescope. Since then its instruments have been subject of a continuous upgrade, making the GTC a competitive telescope for the GRB field. So far (Dec. 2012) the GTC have followed up 25 GRBs, measuring 10 redshifts, and discovering two SNe associated to GRBs. The new generation of instruments foreseen for the coming years will contribute substantially to enhance the knowledge of these puzzling explosions.
\end{abstract}

\section{Introduction}

With its $10.4 \mathrm{~m}$ segmented primary mirror the Gran Telescopio Canarias (GTC) is currently the largest optical telescope of the World. It is privilegedly located at the Observatory of Roque de los Muchachos (La Palma, Spain), several hours in advance with respect to other large aperture telescopes sited at the American continent.

Currently two instruments are mounted on the GTC, OSIRIS and CANARICAM, the second one still being in the commissioning phase. OSIRIS is an imager

\footnotetext{
1 Instituto de Astrofísica de Andalucía (IAA-CSIC), Glorieta de la Astronomía s/n, 18008, Granada, Spain

2 Unidad Asociada Grupo Ciencia Planetarias UPV/EHU-IAA/CSIC, Departamento de Física Aplicada I, E.T.S. Ingeniería, Universidad del PaísVasco UPV/EHU, Alameda de Urquijo s/n, 48013 Bilbao, Spain

${ }^{3}$ Ikerbasque, Basque Foundation for Science, Alameda de Urquijo 36-5, 48008 Bilbao, Spain

4 Dark Cosmology Centre, Niels Bohr Institute, Juliane Maries Vej 30, 2100, Copenhagen $\varnothing$, Denmark

${ }^{5}$ Departamento de Física, Universidad de Jaén, Campus Las Lagunillas, 23071 Jaén, Spain

6 Astronomical Observatory, Nikolaev National University, Nikolaev, Ukraine
} 
and spectrograph for the optical wavelength range $(0.365-1.05 \mu \mathrm{m})$ located in a Nasmyth GTC focus. Its field of view is $7.8 \times 8.5$ arcmin (Cepa et al. 2003). Apart from the standard broad-band imaging and long-slit spectroscopic capability, it provides additional capabilities such as the narrow-band tunable filters (TFs) imaging. The OSIRIS TFs allow to construct filters as narrow as $\sim 10 \AA$, so it is ideal to scan host galaxy fields tuned on the typical emission lines ([O II], [O III] $\left., \mathrm{H}_{\alpha}, \ldots\right)$.

\section{Main results of the GTC observations}

25 GRBs have been followed up spectroscopically or photometrically since April $2009^{7}$. The reaction time of the spectroscopic Target of Opportunity (ToO) observations performed to date range from $\sim 2$ to $\sim 24$ hours. 12 spectroscopic triggers were executed, measuring 10 redshifts, implying a $83 \%$ success rate. Two new SNe were spectroscopically confirmed (GRB 120422A \& GRB 111211A) and two new GRB host galaxies identified (the ones of GRB 101225A and GRB 101219A, both with $r_{A B}^{\prime}>26.5$ ). In addition, the host galaxy of the super-luminous SN 2006oz was also localized (Leloudas et al. 2012). Emission lines of two GRB host fields were detected with the TF (NGC 2770 and the host of GRB 111211A). Here we report a brief summary of the most relevant observations.

\subsection{GRB 090709A}

The observation by our group of this GRB represented the first scientific result of the GTC (Castro-Tirado et al. 2009). The GTC observations were carried out in the $i^{\prime}$-band $\sim 41$ hours after the GRB. The non-detection of any source in the XRT error circle down to $i^{\prime} \sim 25.5$ put constraining limits on the Galactic origin of the source (Cenko et al. 2010).

\subsection{GRB 091127A}

The GTC observed this GRB from 8 to 246 days after the burst in three epochs. The observations were carried in three broad-band filters $\left(r^{\prime} i^{\prime} z^{\prime}\right)$. The data were important to confirm the presence of a supernova (SN 2009nz) bump associated to the GRB. The GTC observations were also relevant to find evidence of a possible jet break in the afterglow lightcurve. The GTC data also provided precious

\footnotetext{
${ }^{7}$ GRB 090404, GRB 090709A (Castro-Tirado et al. 2009), GRB 091202 (de Ugarte Postigo et al. 2009), GRB 100316A, GRB 100418A, GRB 100614A (Guziy et al. 2010), GRB 100816A (Gorosabel et al. 2010a), GRB 101225A (Thöne et al. 2011a), GRB 110328A (Thöne et al. 2011b), GRB 110422A (de Ugarte Postigo et al. 2011a), GRB 110503A, GRB 110801A (Cabrera Lavers et al. 2011), GRB 110918A (de Ugarte Postigo et al. 2011b), GRB 111022B (Gorosabel et al. 2011a), GRB 111117A (Sakamoto et al. 2013), GRB111211A (de Ugarte Postigo et al. 2012), GRB 111228A, GRB 120326A (Tello et al. 2012) GRB 120327A (Sánchez-Ramírez et al. 2012a), GRB 120422A (Sánchez-Ramírez et al. 2012b), GRB 120624B, GRB 120729A, GRB 120811C (Thöne et al. 2012), GRB 120907A (Sánchez-Ramírez et al. 2012c) and GRB 121226A (Castro-Tirado et al. 2012).
} 
information on the host galaxy colours. See Vergani et al. (2011) for a detailed description.

\subsection{GRB 100219A}

This GRB was observed jointly with the VLT $(+\mathrm{XS})$ and GTC. The VLT spectrum measured the metallicity from the afterglow spectrum, whereas the GTC detected a faint host galaxy at a magnitude level of $i^{\prime} \sim 26.7$ (Thöne et al. 2013). These observations are (jointly with the ones of GRB 101225A, see below) one of the deepest observations we have carried out so far with the GTC.

\subsection{GRB 101225A}

GRB 101225A, also known as the Christmas Burst, has been one of the targets most extensively observed by our programs at GTC. The GTC data were crucial to detect the unusual thermal component of its optical emission, which was fitted by a cooling black body spectrum. The GTC also allowed to construct the spectral energy distribution (SED) of its likely supernova (Thöne et al. 2011c). The GTC observations (both spectroscopic and photometric) were carried out from $\sim 2$ to $\sim 180$ days after the gamma-ray emission. The GTC monitoring was based on 5 multi-colour and one spectroscopic visit (grism R300B). A deep late imaging revealed a faint object at $r_{A B}^{\prime}=26.90 \pm 0.14$ which we associate to its host galaxy.

\subsection{GRB 110328A/Sw $1644+57$}

Sw 1644+57, initially named as GRB 110328A, seems not to be related to any stellar death as the previously discussed objects. The GTC data contributed to support the theory that this high-energy event was due to an outburst from a massive black hole in the nucleus of a galaxy.

Two photometric visits were performed in $i^{\prime}$ and $z^{\prime}$ bands 0.7 and 12.7 days after the burst, respectively. In addition $81 i^{\prime}$-band images of 20 -seconds failed to detect rapid optical variability. GTC spectroscopy of Sw $1644+57$ was obtained starting 15.7 hours after the burst. We used the R300B grism with a 1 arcsecond wide slit and a $2 \times 2$ binning, taking three exposures of 1200 seconds. The performance of the OSIRIS spectrograph with respect to Keck(+DEIMOS) and Gemini(+GMOS) was satisfactory, as displayed in Figure 2 of Levan et al. (2011).

\subsection{NGC 2770}

NGC 2770 at a distance of $\sim 27 \mathrm{Mpc}$ hosted two SNe simultaneously, the standard Ib-type SN 2007uy and the more studied SN 2008D, which was uniquely detected as an X-ray burst. In addition a third Supernova (SN 1999eh) occurred in the same galaxy a few years before. Thus, NGC 2770 represents a unique galaxy to compare the explosion sites of standard SNe and the one of a peculiar event like SN 2008D, which has been subject of an intensive debate due to its possible connection with Gamma-Ray Bursts. With this aim, we initiated in May 2010 a $\mathrm{H}_{\alpha}$ imaging 


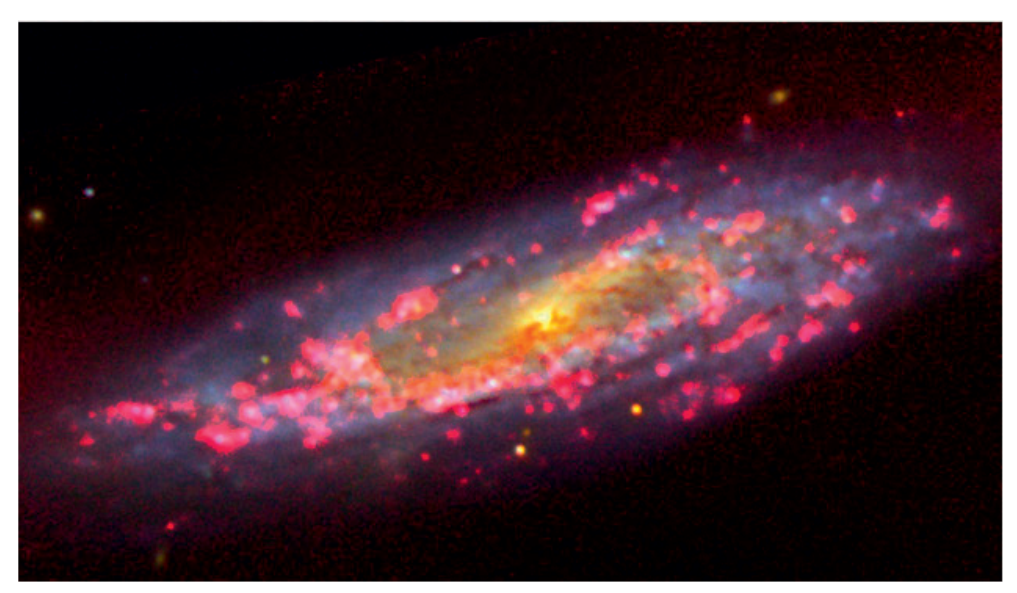

Fig. 1. The image shows the $\mathrm{H}_{\alpha}$ emitting regions (magenta) plotted on the broad-band image taken by VLT for NGC 2770. The narrow-band filter (FWHM $\sim 15 \AA$ ) constructed with the TF produced a high contrast.

campaign using the TF with a width of $15 \AA$ (Gorosabel et al. 2011b). Then the $\mathrm{H}_{\alpha}$ map was correlated with polarization data previously taken for SN 2007uy and SN 2008D with VLT, NOT and $2.2 \mathrm{~m}$ CAHA (Gorosabel et al. 2010b). We estimated the orientation of the interstellar polarization (ISP) at the position of SN 2007uy and, most interestingly, at the site of SN 2008D. The main result is that the electric vector of the ISP was aligned with $\mathrm{H}_{\alpha}$ structures seen at the SN 2008D explosion site (see Gorosabel et al. 2011b for more details). Figure 1 shows the $\mathrm{H}_{\alpha}$ image taken with the GTC over-imposed on the broad-band image taken with VLT.

\section{The future of the GTC}

A different set of instruments are expected to be progressively operative at GTC. In 2013-2014 CANARICAM will be operative. CANARICAM is a mid infrared $(7.5-25 \mu \mathrm{m})$ imager with spectroscopic and polarimetric capabilities. CANARICAM is currently in the commissioning phase and we expect it will be suitable to observe at least nearby host galaxies.

In 2014 the CIRCE camera should arrive in the observatory. CIRCE is a near-IR $(1-2.5 \mu \mathrm{m})$ imager with low-resolution spectroscopic and polarimetric capabilities. CIRCE will be our work-horse for high-redshift, dark GRBs and polarimetric studies. Further, a new generation of instruments is foreseen. Among them we remark MEGARA (an optical high-resolution IFU and MOS) and EMIR (a near-IR widefield medium-resolution multiobject spectrograph), which will be prepared to exploit the observation of bright afterglows, nearby host galaxies and their environments. 
We think that all the above future instruments will contribute to make the GTC even a more efficient telescope for GRB science.

This study was carried out in the framework of the Unidad Asociada IAA-CSIC at the group of planetary science of ETSI-UPV/EHU. This work was supported by the Ikerbasque Foundation for Science.

\section{References}

Cabrera Lavers, A., de Ugarte Postigo, A., Castro-Tirado, A.J., et al., 2011, GCN 12234 Castro-Tirado, A.J., de Ugarte Postigo, A., Gorosabel, J., et al., 2009, GCN 9655 Castro-Tirado, A.J., Jelinek, M., Sánchez-Ramírez, R., et al., 2012, GCN 14114 Cenko, S.B., Butler, N.R., Ofek, E.O., et al., 2010, AJ, 140, 224

Cepa, J., Aguiar-González, M., Bland-Hawthorn, J., et al., 2003, SPIE 4841, 1739 de Ugarte Postigo, A., Castro-Tirado, A.J., Gorosabel, J., et al., 2009, GCN 10247 de Ugarte Postigo, A., Castro-Tirado, A.J., Gorosabel, J., et al., 2011a, GCN 11978 de Ugarte Postigo, A., Gorosabel, J., Castro-Tirado, A.J., et al., 2011b, GCN 12375 de Ugarte Postigo, A., Thöne, C.C., Gorosabel, J., et al., 2012, GCN 12802 Gorosabel, J., Castro-Tirado, A.J., Tanvir, N.R., et al., 2010a, GCN 11125 Gorosabel, J., de Ugarte Postigo, A., Castro-Tirado, A.J., et al., 2010b, A\&A, 522, 14 Gorosabel, J., Cabrera Lavers, A., Sánchez-Ramírez, R., et al., 2011a, GCN 12494 Gorosabel, J., de Ugarte Postigo, A., Castro-Tirado, A.J., et al., 2011b, AdSpR, 47, 1421 Guziy, S., Jelinek, M., Gorosabel, J., et al., 2010, GCN 10967 Leloudas, G., Chatzopoulos, E., Dilday, B., et al., 2012, A\&A, 541, L129 Levan, A.J., Tanvir, N.R., Cenko, S.B., et al., 2011, Science, 279, 1011 Sakamoto, T., Troja, E., Aoki, K., et al., 2013, ApJ, in press [arXiv:1205.6774] Sánchez-Ramírez, R., Gorosabel, J., Castro-Tirado, A.J., et al., 2012a, GCN 13146 Sánchez-Ramírez, R., Leloudas, G., de Ugarte Postigo, A., et al., 2012b, GCN 13281 Sánchez-Ramírez, R., Gorosabel, J., de Ugarte Postigo, A., et al., 2012c, GCN 13723 Tello, J.C., Sánchez-Ramírez, R., Gorosabel, et al., 2012, GCN 13118 Thöne, C.C., Guziy, S., Castro-Tirado, A.J., et al., 2011a, GCN 11568 Thöne, C.C., Gorosabel, J., de Ugarte Postigo, A., et al., 2011b, GCN 11834 Thöne, C.C., de Ugarte Potigo, A., Fryer, C.L., et al., 2011c, Nature, 480, 72 Thöne, C.C., de Ugarte Postigo, A., Gorosabel, J., et al., 2012, GCN 13628 Thöne, C.C., Fynbo, J.P.U., Goldoni, P., et al., 2013, MNRAS, 428, 3590 Vergani, S.D., Flores, H., Covino, S., et al., 2011, A\&A, 535, 127 
